\title{
Correlating Microstructure to in situ Micromechanical Behaviour and Toughening Strategies in Biological Materials
}

Richard E Johnston ${ }^{1 *}$, Ria L Mitchell ${ }^{1}$, Cameron Pleydell-Pearce ${ }^{1}$, Mark Coleman ${ }^{1}$, Laura North ${ }^{1}$, David LaBonte $^{2}$, Michelle Oyen ${ }^{3}$, Rachel Board ${ }^{1}$, Edward C Pope $^{4}$, Hari Arora $^{5}$ and David Howells ${ }^{5}$

1. Advanced Imaging of Materials (AIM) Facility, College of Engineering, Swansea University, Swansea, UK.

2. Department of Bioengineering, Imperial College London, UK.

3. Department of Engineering, East Carolina University, Greenville, North Carolina, USA.

4. College of Science, Swansea University, UK.

5. College of Engineering, Swansea University, Swansea, UK.

* Corresponding author: r.johnston@swansea.ac.uk

Multi-modal and multi-scale imaging and characterization using light, electron, and X-ray microscopy can uncover new insights into diverse hierarchical biological materials. X-ray microscopy $(\mu \mathrm{CT} / \mathrm{XRM})$ reveals previously undiscovered internal microarchitectures, and generates 3D representations of complex surface structures, while light and electron microscopy provide microstructural, chemical, and crystallographic characterisation. In addition, in situ micromechanical deformation can elaborate both the properties of biological materials, and their deformation and fracture behavior. Fusing these techniques together in correlative workflows can extend the fundamental basis of materials science structure/property relationships - to study structure/property/function relationships honed by millions of years of evolution.

Here, we demonstrate the correlative potential of numerous coupled systems at different length scales: X-ray microscopy (XRM), scanning electron microscopy (SEM), nanoindentation, and micromechanical in situ testing to holistically investigate the chemical composition, microstructural and nano/micromechanical properties of a biological mineralised material - the internalized shell, or cuttlebone, of the cuttlefish Sepia officinalis.

The cuttlebone comprises an apical dorsal shield to the top of the organism above a series of chambers separated horizontally by septa, and reinforced by meandering vertical pillars between the septa. Previous work identified discrete microstructural and micromechanical structural units within the cuttlebone [1]; the dorsal shield consists of two stiff and hard layers (outer shield and inner shield) showing prismatic mineral organization encapsulating a more ductile and compliant layer (middle shield) exhibiting a lamellar structure, enriched with organic matter. A similar organization is found in the chambers, which are separated by septa, and supported by the meandering pillars. Like the dorsal shield, septa contain two layers, one with lamellar and one with prismatic organization, which differ significantly in their mechanical properties: layers with prismatic organization are a factor of three stiffer and up to ten times harder than those with lamellar organization [1].

Combining in situ nanoindentation with XRM identifies a possible crack-arresting mechanism in the dorsal shield, arising from its graded multi-layered structure of varying stiffness, hardness, chemistry, mineralization, and microstructure. A Bruker Hysitron Intraspect 360 in situ diamond nanoindenter implemented inside a Zeiss Xradia Versa520 X-ray microscope initiated a crack at the outer surface of the dorsal shield that propagated through the outer prismatic layer, reaching the interface with the middle laminar region, but terminating there (Fig.1). 
Similar crack arresting behavior has been observed in the dentin-enamel junction in human teeth [2] and explained as crack deflection at the interface between dissimilar elastic materials [3]. Other biological materials with periodic varying Young's Modulus, albeit at a much smaller scale, including nacre (mother of pearl) [4], and in the silica skeleton of hexactinellid sponges [5], have also demonstrated crack arresting properties. Identifying these mechanisms for arresting cracks in nature can inform design processes for a new generation of tough, layered materials in engineering [6].

\section{References:}

[1] L North et al., APL Materials 5 (2017), p. 14.

[2] V Imbeni et al., Nature Materials 4 (2005), p. 229.

[3] H Ming-Yuan and J.W. Hutchinson, Journal of Solids and Structures 25(9) (1989), p. 1053.

[4] P Fratzl et al., Adv. Mater. 19(18) (2007), p. 2657.

[5] J Aizenberg et al., Science 309(5732) (2005) p. 275.

[6] The authors would like to acknowledge the financial support of the Engineering and Physical Sciences Research Council (EPSRC) Grant No. EP/M028267/1, and the European Social Fund (ESF) through the European Union's Convergence programme administered by the Welsh Government.
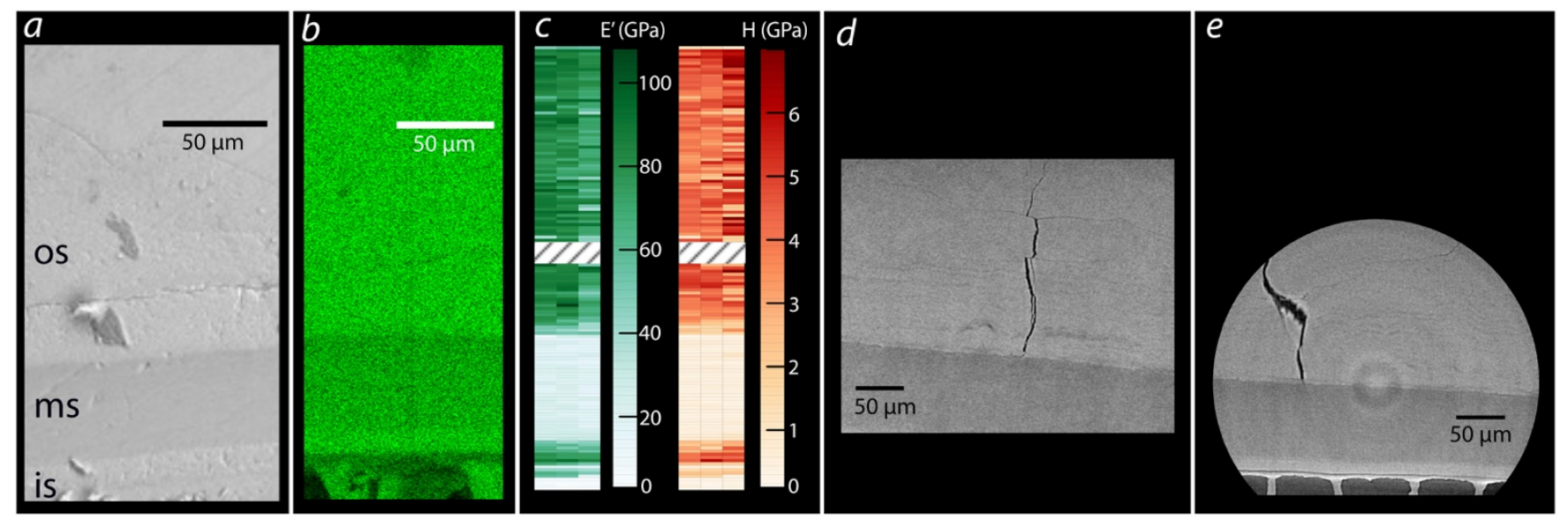

Figure 1. Each pane (a-e) represents the cuttlebone dorsal shield in cross section with imaged/characterized with different modalities. (a) Backscatter scanning electron microscopy image highlighting a change in atomic mass across the layers. (b) Energy dispersive spectroscopy of calcium distribution. (c) Nanomechanichal mapping. Distinct differences in indentation modulus, E', and indentation hardness, $\mathrm{H}$, between the layers with the accelerated property mapping feature of the 950 Triboindenter. The area crossed-out with diagonal lines is an unmapped region. (d \& e) X-ray microscopy of the dorsal shield, visualised as 2D slices oriented perpendicular to each other. The outer surface of the shield is oriented to the top of the slices. A crack can be seen extending through the uppermost layer, arresting at the interface with the middle layer of the dorsal shield. os-outer shield, ms - middle shield, is - inner shield. 